\title{
CARNAVAL: Mascote para o Aprendizado Virtual de Língua Portuguesa
}

\author{
Mario Figueiró Zemor ${ }^{1}$, Alan Velasques Santos ${ }^{1}$, \\ Antonio Silva Sprock ${ }^{2}$, Rosa Maria Vicari ${ }^{1}$ \\ ${ }^{1}$ Instituto de Informática - Universidade Federal \\ do Rio Grande do Sul (UFRGS) - Porto Alegre - RS - Brazil \\ ${ }^{2}$ Universidad Central de Venezuela, Facultad de Ciencias, Escuela de Computación \\ Av. Los Ilustres, Los Chaguaramos, Caracas, 1043, Venezuela. \\ mario.ufrgs.infegmail.com, avsantoseinf.ufrgs.br, \\ antonio.m.silvaducv.ve, rosadinf.ufrgs.br
}

\begin{abstract}
The article shows the development of a Learning Object based on a virtual mascot (PET), called CARNAVAL, for learning Brazilian Portuguese by mobile devices. The application has its dynamics developed through a game (learning game) which proposes the user the task of caring for a virtual PET. The implementation is based on a client-server architecture, it was developed using Bootstrap framework and the methodology is Agile Model Driven Development (AMDD), developed by Scott Ambler.
\end{abstract}

Keywords: Learning Object, Virtual Mascot, PET, Brazilian Portuguese Language.

Resumo. $O$ artigo mostra o desenvolvimento de um Objeto de Aprendizagem baseado em um mascote virtual (PET), chamado CARNAVAL, para o aprendizado de língua portuguesa brasileira via dispositivos móveis. $O$ aplicativo tem sua dinâmica desenvolvida através de um jogo (game learning) que propõe ao usuário a tarefa de cuidar de um mascote virtual. A implementação é baseada em uma arquitetura Cliente-Servidor, foi desenvolvida usando o framework Bootstrap $e$ a metodologia de Desenvolvimento Ágil com Base em Modelos (AMDD), desenvolvida por Scott Ambler.

Palavras Chave: Objeto de Aprendizagem, Mascote Virtual, PET, Língua portuguesa brasileira.

\section{Contexto}

A educação está mudando. Professores e alunos estão sendo desafiados a desenvolver novas técnicas de educação diferentes das que existiam no século passado. A demanda necessária para propiciar uma educação apropriada para a geração $\mathrm{Z}^{1}$ não poderá ser atendida sem considerar as características desta população específica (Lima et. al, 2014).

1 Definição sociológica para definir a geração de pessoas nascidas a partir dos anos 90 até os dias de hoje.

DOI: $10.5753 /$ cbie.wcbie.2015.778 


\section{CBIE-LACLO 2015}

Anais dos Workshops do IV Congresso Brasileiro de Informática na Educação (CBIE 2015)

Segundo Prensky (2001) os chamados nativos digitais (geração Z) nasceram em um mundo rodeado por tecnologias digitais utilizando-as, desde a infância, o que os fez desenvolverem habilidades avançadas no reconhecimento de padrões digitais, utilizando facilmente os recursos de comunicação e serviços de internet. Este fato deixa o nativo desta geração acostumado a ter várias fontes de estímulos multimídia com alta interatividade.

Nos últimos anos, com o crescimento dos dispositivos móveis conectados à internet, passamos a viver um novo começo dentro da antiga era digital, a era da mobilidade e da computação ubíqua. Nesta cada simples elemento ao nosso redor está conectado na internet enviando e recebendo dados e interagindo com os usuários. Os usuários aprenderam e passaram a depender desta tecnologia para facilitar as suas vidas.

Estudos recentes (Adsmovil, 2014; GuiaLocal, 2014) afirmam que a utilização de smartphones na América Latina cresceu, rapidamente, em 2014, superando o uso de computadores tradicionais. Junto a esse grande crescimento, surge, também, a necessidade de mudança em diversas áreas, destacando entre elas o setor da educação.

A UNESCO, em seu documento que apresenta normas de competências para professores em TICs (Tecnologias de Informação e Comunicação), apoia professores a desenvolverem competências para proporcionar um ensino de melhor qualidade para seus alunos fazendo uso de tecnologias, inclusive, salienta que os profissionais da área de ensino devem estar preparados para utilizar a tecnologia e saberem como dar suporte ao ensino por meio desta.

Com esses avanços nas Tecnologias de Informação e Comunicação (TICs), vieram os objetos de aprendizagem (OA) (Hernández; Silva, 2013), métodos (Silva; Ponce; Hernández, 2013), softwares para o desenvolvimento (Silva; Flores; Rebete, 2015) e, mais tarde, os Recursos Educacionais Abertos (REA), que oferecem grandes oportunidades para o apoio à educação e satisfação dos seus usuários (Velásquez et. al, 2014).

O tempo, nos dias de hoje, se tornou fundamental para a geração $\mathrm{Z}$ que precisa estar, constantemente, aprendendo sobre os mais diversos assuntos. Entretanto, surgem dois questionamentos com o levantamento desta problemática: é possível aprender sem ter tempo e, além disso, há a possibilidade de frequentar aulas presenciais ou mesmo utilizar um computador de mesa enquanto se vive cercado de informações e distrações fornecidas pelos mais diversos meios durante todo o cotidiano.

Segundo Collins (2005), o aprendizado móvel via aparelhos celulares se tornou a única resposta para esses questionamentos. Collins ainda cita em seu artigo que o aprendizado de língua estrangeira é um dos aprendizados mais requisitados e necessários nos dias de hoje, em virtude da globalização. Com isso, Collins sugere um método para o ensino de línguas via celular, no qual o usuário não precisa ficar ligado a um computador de mesa ou mesmo ir a um curso presencial.

Segundo Conole (2002), PETs (Peripatetic Electronic Teachers) fornecem e disponibilizam os meios para um agente autônomo atuar como professor no mundo digital, e esta visão do agente como professor ainda pode ser muito explorada. Em seu artigo, Conole faz um levantamento sobre trabalhos que estão desenvolvendo estes agentes autônomos e, ainda, encontra um grande crescimento neste estilo de PETs com o passar dos anos. 
CBIE-LACLO 2015

Anais dos Workshops do IV Congresso Brasileiro de Informática na Educação (CBIE 2015)

\section{CARNAVAL}

No referido contexto, foi implementada uma solução, utilizando OAs, que consiste em um mascote (PET) virtual para o aprendizado de língua portuguesa via dispositivos móveis, de tal forma que usuário não precisará ficar limitado apenas à utilização de um computador de mesa ou estar presente em uma sala de aula, visando assim uma busca pelo conhecimento através de dispositivos móveis além dos limites escolares.

O CARNAVAL possui uma grande quantidade de informações que são referentes ao conteúdo pedagógico de ensino dividido por níveis, sendo assim associase à cada nível alcançado pelo usuário uma gama de informações referentes à temática vigente, ou seja, a cada novo nível atingido, são adicionadas imagens e itens que fazem parte do novo contexto.

Todo este conteúdo tornaria o aplicativo, extremamente, grande em questão de tamanho, o que dificultaria a sua distribuição para o cliente em vários casos, tais como: as lojas de aplicativos limitam seus tamanhos máximos, o uso em dispositivos de menor capacidade ficaria comprometido, e, por fim, o download do aplicativo em planos de internet mais básicos seria muito custoso e, talvez inviável, pois a informação teria que ser enviada ao cliente de uma só vez.

A metodologia de ensino tem como objetivo realizar o repasse do conhecimento através de testes diários que são realizados pela interação com o mascote, porém de maneira indireta deixando o aprendizado transparente ao usuário. O ensino se dará durante as interações do usuário com o mascote, no qual, a cada interação, o usuário estará vivendo na pele do mascote futuras situações cotidianas que enfrentará ao imergir no português.

\subsection{Arquitetura utilizada}

Com o propósito de solucionar esses casos, é proposta a implementação do aplicativo baseada em uma arquitetura Cliente-Servidor. Um servidor foi desenvolvido, no qual estão contidas todas as informações necessárias para a atualização do aplicativo (conteúdo pedagógico, itens e imagens). Isto pode ser visto na Figura 1 que representa a arquitetura do sistema.

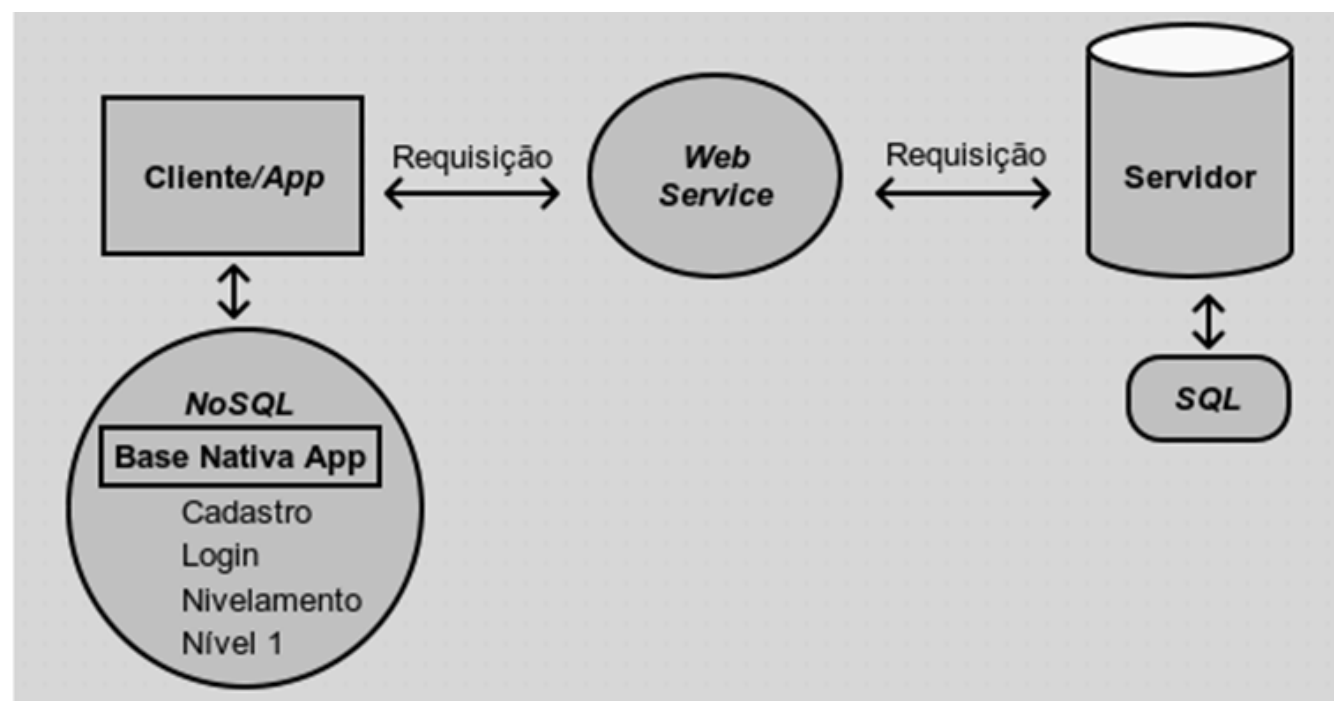

Figura 1. Arquitetura do sistema proposto. 
Cada solicitação possui um papel fundamental no desempenho e continuidade do aplicativo, tendo em vista que são realizadas atualizações conforme a necessidade real que o usuário possui de avançar e, consequentemente, realizar uma conversação com o servidor. O aplicativo solicitará novas informações para reabastecer o banco local NoSQL com informações necessárias para o novo nível do usuário. As informações locais são enviadas para o Servidor, através de um Web Service que realiza o passo intermediário na troca de informações entre o cliente e o servidor.

Podemos exemplificar a situação apresentada acima, utilizando como base uma passagem de nível do usuário. Quando esta ação ocorre, uma solicitação é feita ao Web Service enviando as informações atuais do nível e requerendo novas informações referentes ao novo nível atingido. Uma vez recebida a mensagem, o Web Service comunica-se com o Servidor, através de um protocolo, então o servidor realiza a busca da informação solicitada no banco de dados SQL e retorna ao Web Service que, por sua vez, repassa as informações obtidas ao aplicativo, podendo, assim, aplicar as modificações necessárias para o progresso do jogo.

Ao instalar o aplicativo diretamente da loja de seu sistema operacional, o usuário recebe apenas as informações essenciais que possibilitam a realização de tarefas iniciais como o nivelamento e o nível básico que estão contidas no banco local NoSQL. Posteriormente, novos dados são solicitados ao servidor conforme o progresso do estudante.

Em outras palavras, esse pacote de tecnologias, implementadas em conjunto, permite a maior organização e facilidade na transferência de dados quando há uma requisição, viabilizando alterações de parte ou todo aplicativo, pois não geram nenhuma modificação de imediato em qualquer dispositivo que esteja utilizando a última versão. A requisição atualiza, somente, aquilo que é essencial para completar os próximos níveis de estudo.

\subsection{Metodologia}

A metodologia ágil Ambler (2002), chamada Desenvolvimento Ágil com Base em Modelos (Agile Model Driven Development, AMDD), é uma metodologia iterativa e incremental, na qual são desenvolvidos modelos ágeis e simples utilizados para desenvolver o sistema.

A primeira fase, chamada iteração 0 , é apresentada pela caixa verde (Figura 2), integrado duas atividades: as necessidades iniciais e a arquitetura original. Isto é, não é realizada uma descrição completa do desenvolvimento, pois este se dá de forma incremental, no qual as necessidades iniciais são implementadas e analisadas. Após a análise, são criados novos requisitos (se necessário) e é gerada uma nova iteração, desta forma, quaisquer alterações necessárias na iteração seguinte não comprometerão o trabalho realizado até o momento.

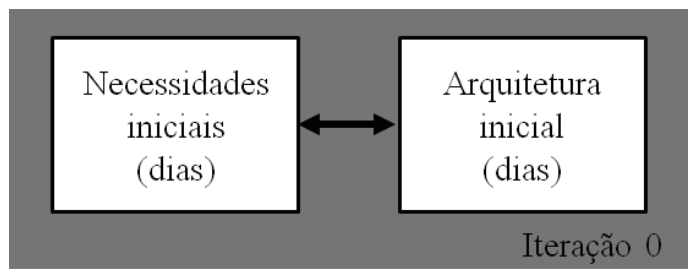

Figura 2: Iteração - Ambler (2002) 
CBIE-LACLO 2015

Anais dos Workshops do IV Congresso Brasileiro de Informática na Educação (CBIE 2015)

As atividades restantes (Figura 3): modelagem iteração, chuva de modelos e desenvolvimento/teste, foram executadas em todas as iterações, nas quais o progresso é validado com especialistas, de forma que a chuva de modelos pode ajudar a compreender melhor a necessidade real de funcionalidades que precisamos, passando, diversas vezes, por modelos com diferenças entre si. Através disso, são analisados os benefícios de cada modelo, em particular, para chegar em um modelo final.

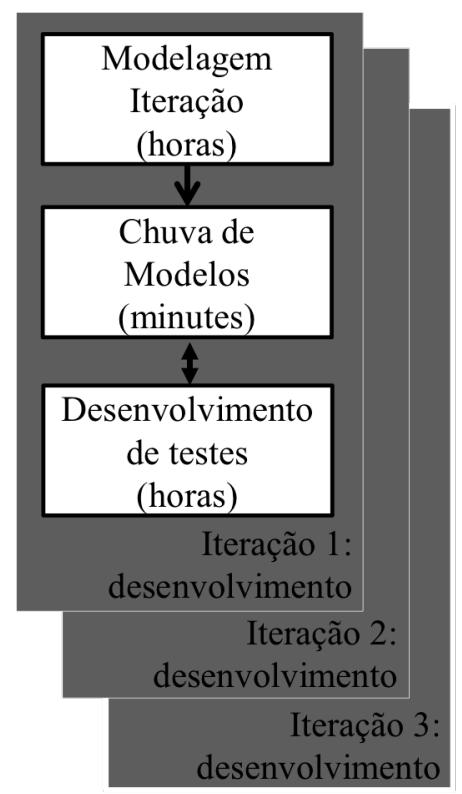

Figura 3: Iterações depois de Iteração 0 da AMDD - Ambler (2002)

\section{Tecnologias utilizadas}

A interface do aplicativo proposto foi projetada visando a usabilidade em dispositivos móveis, fazendo uso de características responsivas, abrangendo, assim, um escopo maior de usuários com diferentes dispositivos. Buscando satisfazer essas propriedades, o sistema foi implementado, a partir da união de tecnologias como o HTML5, o CSS3 e JavaScript (Figura 4).

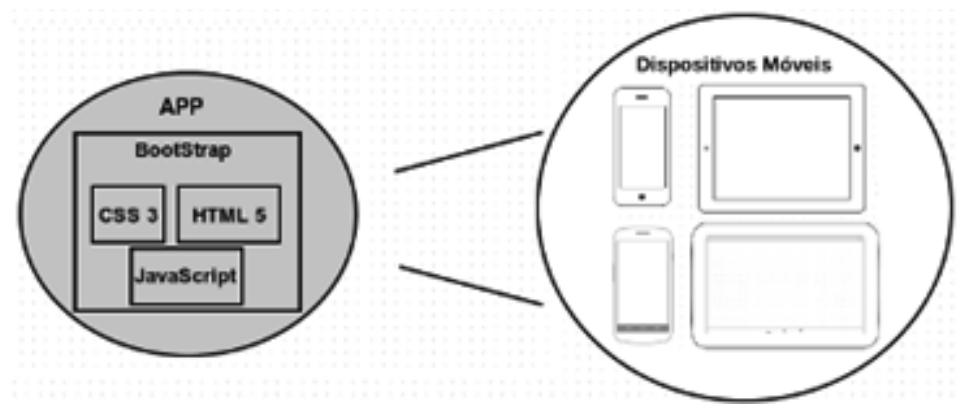

Figura 4. Tecnologias utilizadas.

Em busca de uma maior compatibilidade de visualizações entre diferentes sistemas, foi utilizado o framework Bootstrap, que possui um grande conjunto de funcionalidades prontas implementadas com as tecnologias HTML5, CSS3 e JavaScript. Esse conjunto de tecnologias nos permite adaptar o aplicativo, de acordo com cada dispositivo utilizado pelo usuário, automaticamente, sem as necessidades de duplicação de código. Uma vez que a versão for implementada, a mesma pode ser distribuída diretamente para diversas plataformas diferentes. 
CBIE-LACLO 2015

Anais dos Workshops do IV Congresso Brasileiro de Informática na Educação (CBIE 2015)

\subsection{Interface}

A tela inicial, que pode ser vista na Figura 5, permite ao usuário conectar-se ao jogo através de login e senha. Caso o usuário ainda não possua acesso, ele pode efetuar um cadastro simples e liberar o seu acesso à plataforma. Na tela de cadastro, que pode ser vista na Figura 6, são solicitadas algumas informações básicas ao usuário como nome, e-mail, senha e país. Ainda na Figura 6, podemos observar as outras formas de cadastro que podem ser realizadas a partir do Facebook ou da conta Google do usuário.

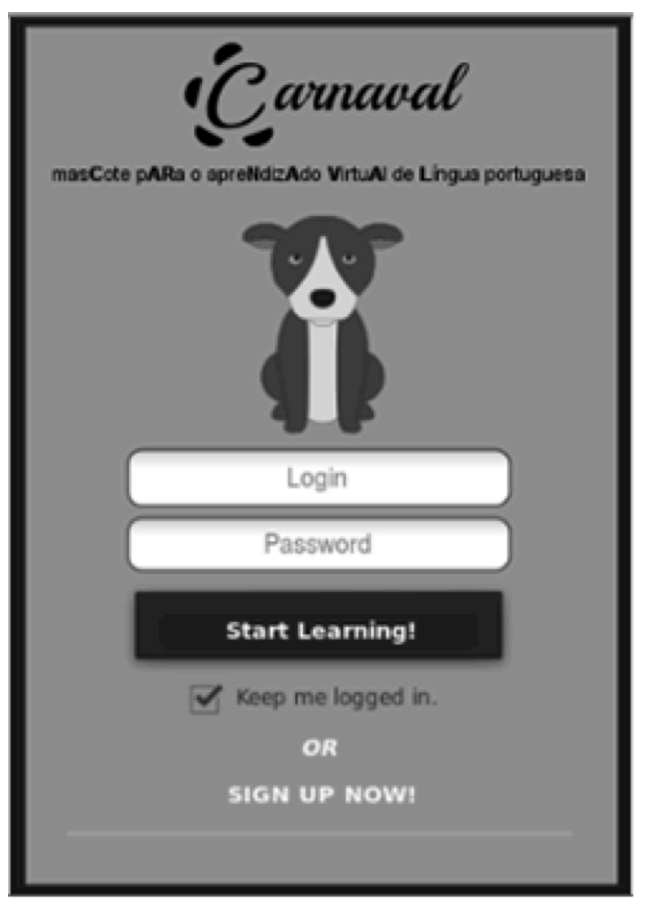

Figura 5. Tela inicial do CARNAVAL.

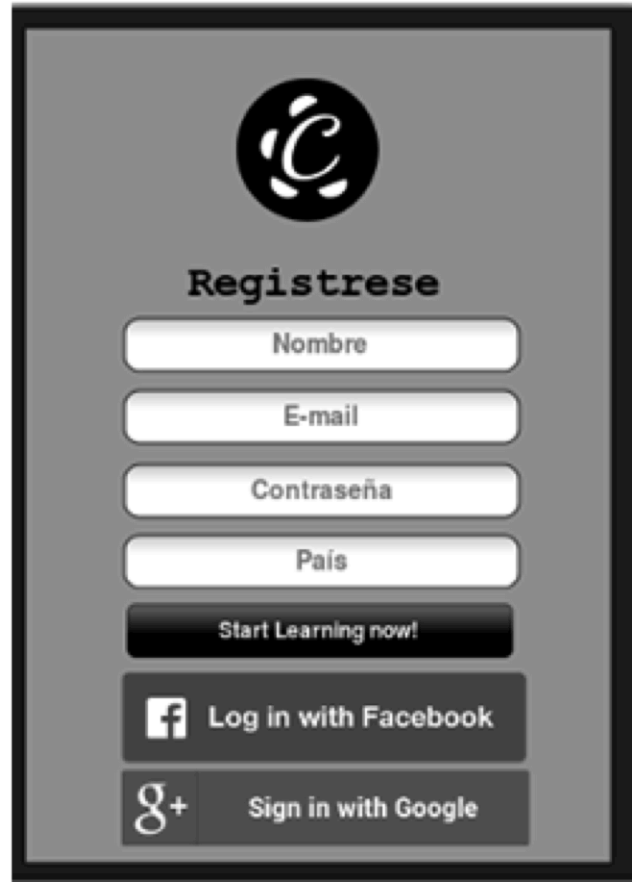

Figura 6. Tela de cadastro.

Após o cadastro, como pode ser visto na Figura 7, o usuário pode escolher entre começar como iniciante, no qual seus estudos iniciam a partir do nível 1, ou realizar um teste de nivelamento, que permite verificar qual o nível de conhecimento do usuário em língua portuguesa, sendo encaminhado para níveis diferentes de acordo com o resultado, como pode ser visto na Figura 8. 
CBIE-LACLO 2015

Anais dos Workshops do IV Congresso Brasileiro de Informática na Educação (CBIE 2015)

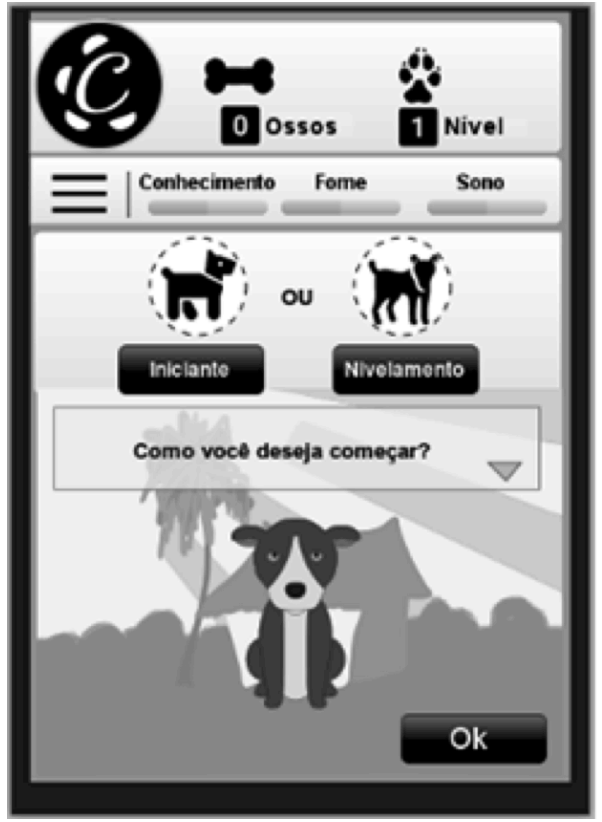

Figura 7. Nivelamento

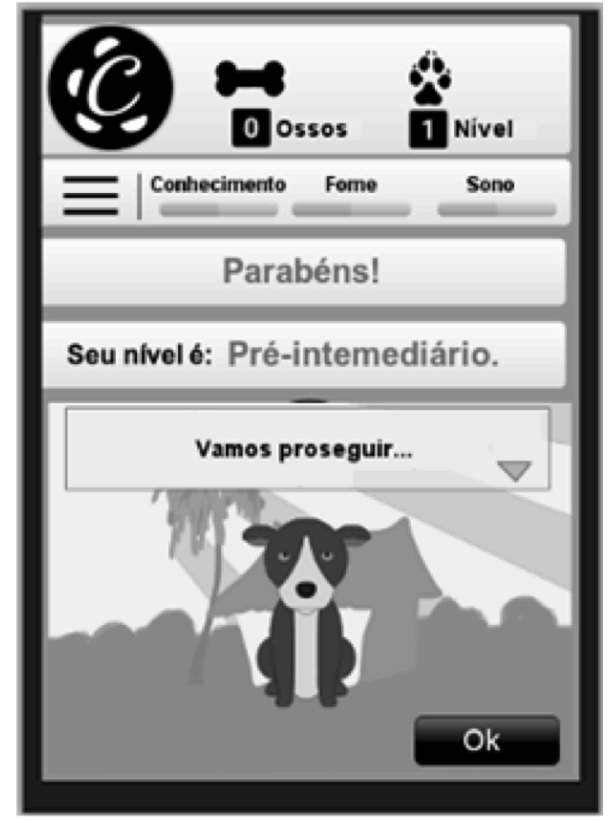

Figura 8. Níveis do PET.

Na Figura 9 observa-se que se for a primeira vez que o usuário faz login, o ambiente irá solicitar um nome para o PET que acompanhará o usuário por um tour, que pode ser visto na Figura 10. Este apresentará as funcionalidades do sistema, tendo por objetivo esclarecer possíveis dúvidas sobre quaisquer funcionalidades.

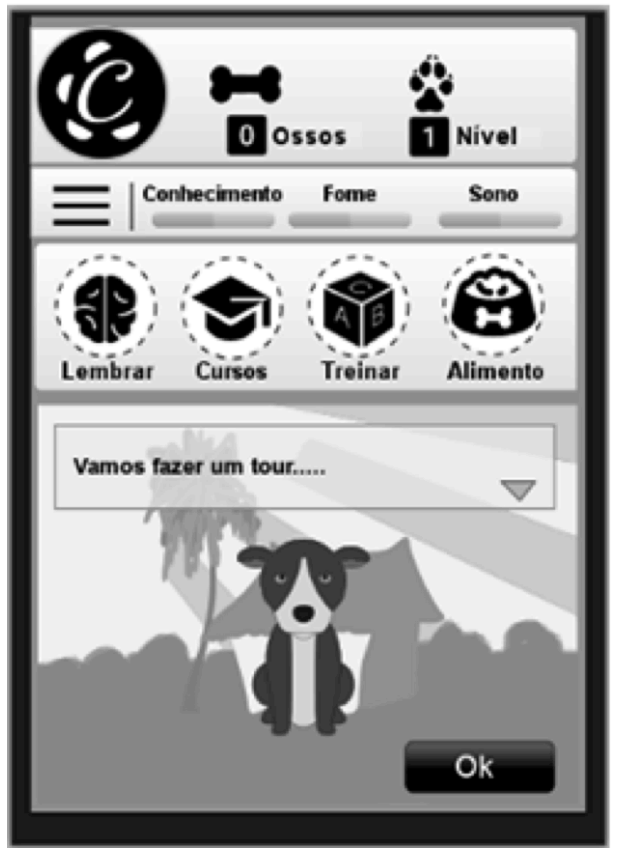

Figura 9. Tela inicial da primeira vez.

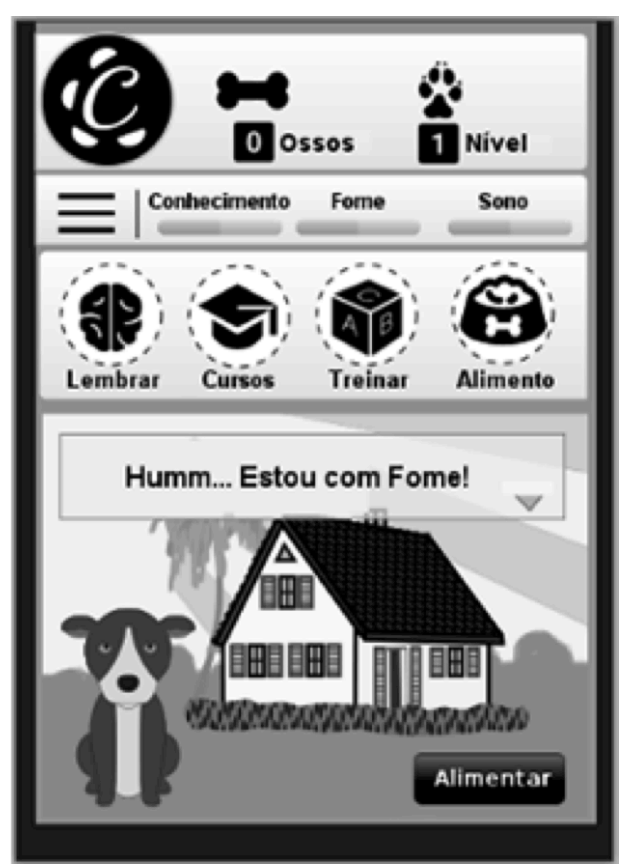

Figura 10. Funcionalidades do PET.

\section{Conclusões e trabalhos futuros}

A geração $\mathrm{Z}$ exige aplicações digitais que são compatíveis com os seus hábitos tecnológicos. O uso de smartphones e dispositivos móveis está aumentando e já ultrapassa o uso de computadores tradicionais, analisando este fato foi encontrada a necessidade de criação de OAs que acompanhem essa mudança, estando disponíveis a qualquer usuário garantindo, também, o fácil acesso em dispositivos móveis. 


\section{CBIE-LACLO 2015}

Anais dos Workshops do IV Congresso Brasileiro de Informática na Educação (CBIE 2015)

Foi desenvolvido um OA baseado em uma mascote virtual (PET), chamado CARNAVAL, para o aprendizado de língua portuguesa através de dispositivos móveis, almejando uma melhoria significativa no modo com que a língua portuguesa é ensinada. O framework Bootstrap foi capaz de atender as necessidades de desenvolvimento de recursos do OA, de modo que através do mesmo foi possível, de forma eficiente ,abranger um grande número de dispositivos diferentes. $\mathrm{O}$ uso da metodologia ágil foi fundamental para o desenvolvimento e conclusão do aplicativo devido ao grande número de alterações e adaptações feitas para atingir um número maior de usuários e aumentar a capacidade do aprendizado do aluno com funcionalidades mais eficientes que foram testadas em cada fase do desenvolvimento.

O CARNAVAL será testado com estudantes venezuelanos da Universidade Central da Venezuela, em um futuro próximo. Estará disponível em lojas de aplicativos de tablets e smartphones. Seguindo esses passos, uma maior visibilidade e valorização da língua portuguesa será atingida, pois é esperado que o CARNAVAL seja referência para estrangeiros que buscam o conhecimento desse idioma e, também, para futuros trabalhos na área de desenvolvimento de softwares para o ensino de línguas.

\section{Referências}

Adsmovil (2014). La penetración móvil y el uso de smartphones crecen en América Latina. Disponível em: http://www adsmovil.com/la-penetracion-movil-y-el-uso-desmartphones-crecen-en-america-latina/. Acesso em 15 de julho de 2015.

Ambler, S. (2002) Agile Modeling: Effective Practices for Extreme Programming and the Unified Process. Disponível em: http://www.agilemodeling.com/essays/introductionToAM.htm. Acesso em 16 de janeiro de 2015.

Collins, T. G. (2005). English Class on the Air: Mobile Language Learning with Cell Phones. Proceedings of the Fifth IEEE International Conference on Advanced Learning Technologies.

Conole, G. (2002). The Evolving Landscape of Learning Technology. Research in Learning Technology.

GuiaLocal (2014). Marcada caída del uso de computadoras en Latinoamérica. Disponível em: http://guialocal.com/blog/es/2015/01/12/marcada-caida-del-uso-decomputadoras-en-latinoamerica/. Acesso em 15 de julho de 2015.

Hernández, Y., Silva Sprock, A. (2013). Una Metodología Tecnopedagógica para la Construcción Ágil de Objetos de Aprendizaje Web. Revista Opción. Universidad del Zulia. Vol.29, Num 70. Pp. 66-85. ISSN: 1012-1587. Disponível em:: http://200.74.222.178/index.php/opcion/article/view/6602/6590. Acesso em 12 de maio de 2015.

Kaul, N., Morankar, T., Lolge, S., Gore, S. (2015). Multilingo Android Based Language Learning Mobile Application Via Gaming. IJSART - Volume 1 Issue 4.

Lima, J. V., Singo, F., Filho, A. C., Müller, T., Siva, F. (2014). Objetos de Aprendizaje Multimodales: Diseños y Aplicaciones. Barcelona: UOC, 196 pg.

Microsoft, Service Broker (2015). Disponível em: https://technet.microsoft.com/ptbr/library/ms166049\%28v=sql.105\%29.aspx. Acesso em 12 de maio de 2015. 


\section{CBIE-LACLO 2015}

Anais dos Workshops do IV Congresso Brasileiro de Informática na Educação (CBIE 2015)

Prensky, M. (2001). Nativos Digitais: Imigrantes Digitais. De On the Horizon: NCB University Press, Vol. 9 No. 5.

Silva Sprock, A., Ponce Gallegos, J., Hernández, Y. (2013). Estado del Arte de las Metodologías para el Desarrollo de Objetos de Aprendizaje. Proceedings da VIII Conferencia Latinoamericana de Objetos de Aprendizaje y Tecnologías para el Aprendizaje (LACLO2013). Valdivia, Chile: Universidad Austral de Chile, 21-25 de outubro de 2013. Pp.115-126. ISSN: 1982-1611. Disponível em: http://www.laclo.org/papers/index.php/laclo/article/view/83/77. Acceso 12 de maio de 2015.

Silva Sprock, A., Flores Vitelli, I., Rebete Guillermo, O. (2015). Gestor de Objetos de Aprendizaje Abiertos: GesOA2. Revista Electrónica de Investigación y Docencia (REID). Num. 13, Pp. 135-152. ISSN: 1989-2446. Disponível em: http://revistaselectronicas.ujaen.es/index.php/reid/article/view/1366/1944. Acceso 12 de maio de 2015.

Velásquez Amador, C., Álvarez Rodríguez, F., Muñoz Arteaga, J., Cardona Salas, P., Silva Sprock, A., Hernández, Y., Cechinel, C. (2014). Un Estudio de la Satisfacción Obtenida con el Uso de Objetos de Aprendizaje. Proceedings da IX Conferencia Latinoamericana de Objetos de Aprendizaje y Tecnologías para el Aprendizaje (LACLO2014). Universidad Nacional de Colombia, Manizales, Colombia. 20-24 de outubro de 2014. ISSN: 1982-1611. Disponível em: http://www.laclo.org/papers/index.php/laclo/article/view/253/235. Acceso 12 de maio de 2015. 